\title{
Scheduling of Multimedia Traffic in Interference-Limited Broadband Wireless Access Networks
}

\author{
Mohamed H. Ahmed, Halim Yanikomeroglu, Samy Mahmoud, David Falconer \\ Broadband Communications and Wireless Systems (BCWS) Centre, \\ Dept. of Systems and Computer Engineering, Carleton University, \\ 1125 Colonel By Drive, Ottawa, Ontario, Canada \\ $\{\mathrm{mhahmed} / \mathrm{halim} / \mathrm{mahmoud} / \mathrm{ddf}\} @$ sce.carleton.ca
}

\begin{abstract}
This paper proposes a transmission scheduling algorithm for interference management in broadband wireless access networks. The algorithm aims at minimizing the cochannel interference using basestation coordination while still maintaining the other quality of service $(Q o S)$ requirements such as packet delay, throughput, and packet loss. The interference reduction is achieved by avoiding (or minimizing) concurrent transmission of potential dominant interferers. Dynamic slot allocation based on traffic information in other cells/sectors is employed. In order to implement the algorithm in a distributed manner, basestations have to exchange traffic information. Both real-time and non-real-time services are considered in this work. Results show that significant enhancement in the signal to interference ratio can be achieved which decreases the packet error rate by almost one order of magnitude at the expense of a higher but acceptable packet delay at high loading.
\end{abstract}

\section{Keywords}

Transmission scheduling in wireless networks, broadband wireless access, multimedia over wireless.

\section{INTRODUCTION}

Broadband wireless access networks are considered as the most promising candidate for multimedia services provisioning for residential and small business areas. Multimedia services including real-time traffic (voice and video) and non-real-time traffic (http and ftp data) are to be supported.

Since most of the multimedia traffic is inherently bursty, packet-level performance has to be investigated to ensure that the stringent quality of service (QoS) requirement of most multimedia applications can be met. There are many factors and parameters that affect the QoS in multimedia applications, but the most important one is the transmission scheduling since it has a direct impact on the delay, throughput and signal quality performance.

Numerous algorithms have been proposed for multimedia scheduling over wireless links. Most of these algorithms are in essence modified versions of some scheduling algorithms employed in wireline networks used to cope with the lower transmission rate and high error rate encountered in wireless environment, (see for example [1][3]). Then, several algorithms have proposed the concept of user diversity by making use of the channel variations and allocate a lot of resources for users with good channel conditions and lower (or even no resources at all) to users with bad channel conditions [4]-[6]. These scheduling techniques have been studied either in isolated cells or in multiple cells but without considering the scheduling technique in the interference management. The use of packet scheduling for interference management has been proposed in [7]-[9]. In [7], a new technique has been proposed for time sharing between sectors using time reuse to avoid sources of major interference. An enhanced version has been proposed in [8] by providing different degrees of concurrent transmission in different time slots. However, the main drawback in [7] and [8] is the static nature of the time allocation technique, which can cause resource wasting particularly with bursty traffic sources of multimedia services. In [9], interference management is achieved using basestation (BS) coordination assuming that each BS knows in advance the transmission schedule of other BSs. This assumption is hard to implement in reality.

In this paper, we propose a dynamic time slot allocation that minimizes the cochannel interference. By taking the cochannel interference into account in multiple cells, the scheduling problem becomes more challenging since the transmission scheduling has to consider not only the packet delay and throughput performance but also the signal quality in terms of the signal to interference ratio. BSs exchange information about the available traffic. Then, each BS schedules its local traffic based on this information. Concurrent transmission by potential dominant interfering users is avoided (or at least minimized) by assigning different time slots to those users.

The rest of the paper is organized as follows. Section II presents the systems model. The proposed algorithm is described in section III. Performance measures and Results are presented in section IV. Finally section V contains the summary and conclusions.

\section{SYSTEM MODEL}

A hexagonal cellular structure with a wraparound structure is used in the simulation. Each cell is divided into 6 sectors. Sinc-shaped beam pattern with $60^{\circ}$ beamwidth is used at both BSs and subscriber stations (SSs). While the BS antenna beams are fixed, it is assumed that the antenna beams at the SSs are electronically steered to point at the direction of serving BSs. 
The channel model consists of an exponential path loss model with an exponent $(n)$ of 3 , lognormal shadowing with a standard deviation $(\sigma)$ of 8 , and flat Rayleigh fading. Shadowing samples are spatially correlated with a correlation coefficient of 0.5 for $1 \mathrm{~m}$ displacement. Temporally correlated Rayleigh fading samples are generated using rounded (bell-shaped) Doppler spectrum with a $3-d B$ frequency of $2 \mathrm{~Hz}$ [10]. The Rayleigh fading samples of a user from different BSs are mutually independent.

A frequency reuse plan of $1 / 6$ is employed such that the total spectrum is divided into 6 equal sub-bands allocated to the 6 sectors and reused in each cell as shown in Fig. 1. The employment of directional antennas at both BSs and SSs enables such a tight frequency reuse plan.

Time is divided into frames with a frame-duration of $5 \mathrm{~ms}$ consisting of 9 slots in a TDMA fashion. This work focuses on the downlink performance since it is the limiting factor in many multimedia services. However, the proposed algorithm can be implemented in the uplink as well.

Two services are considered in this study, namely, video service as an example of real-time services and Internet (HTTP/TCP and FTP) traffic as an example of non-realtime services. Packets are generated using $n$-Interrupted Poisson Processes (IPP). The traffic model used in this work is proposed for broadband wireless access networks in [11]. For the Internet traffic, one IPP is used to model the traffic with on and off sojourn times following exponential distribution with transition rates $R_{\text {on_off }}$ and $R_{\text {off } \text { on }}$ respectively. For the video service, two IPPs are used with on and off sojourn times following Pareto distribution with parameters $\alpha_{1}$ and $\alpha_{2}$ respectively. The parameters of the traffic model are listed in Tables 1 and 2 [11]. The superposition of the $n$ Interrupted Poisson Processes models the self-similar traffic of different multimedia services. The targeted bit error rates (BER) are $10^{-4}$ and $10^{-6}$ for the video service and data service, respectively. The maximum delay $\left(D_{\max }\right)$ for video service is $200 \mathrm{msec}$. No maximum delay is specified for the data service.

16-QAM with bit-interleaved coded modulation (BICM) [12] is used in this work. The required SIR values corresponding to the targeted BER levels mentioned above are $10.93 \mathrm{~dB}$ and $9.25 \mathrm{~dB}$ for the video service and data service, respectively.

Fixed users assumed in this work. However, the proposed algorithm can be utilized by nomadic users or even by mobile users. It is assumed that users are uniformly distributed and are assigned to the best serving BS (not necessarily the nearest one).

Table 1. Traffic model parameters of the Internet service

\begin{tabular}{|c|c|c|c|}
\hline IPP\# & $\begin{array}{c}\text { Packet } \\
\text { Arrival Rate } \\
\text { (packet/s })\end{array}$ & $\begin{array}{c}\mathrm{n} \text { to off } \\
\text { Transition } \\
\text { Rate } \\
\left(R_{\text {on_off }} \sec ^{-1}\right)\end{array}$ & $\begin{array}{c}\text { Off to On } \\
\text { Transition } \\
\text { Rate } \\
\left(R_{\text {off_on }} \sec ^{-1}\right)\end{array}$ \\
\hline $\mathrm{IPP}_{1}$ & 22.79 & 0.194 & 0.1455 \\
\hline
\end{tabular}

Table 2. Traffic model parameters of the video service

\begin{tabular}{|c|c|c|c|}
\hline $\mathrm{IPP} \#$ & $\begin{array}{c}\text { Packet } \\
\text { Arrival Rate } \\
\text { (packet/s) }\end{array}$ & $\alpha_{1}$ & $\alpha_{2}$ \\
\hline $\mathrm{IPP}_{1}$ & 112.38 & 1.14 & 1.22 \\
\hline $\mathrm{IPP}_{2}$ & 154.75 & 1.54 & 1.28 \\
\hline
\end{tabular}

It is assumed that the system is interference-limited. Hence, noise is neglected and only cochannel interference is considered.

\section{ALGORITHM DESCRIPTION}

The proposed algorithm schedules the packet transmission in each sector taking into account the traffic information in the sectors of potential dominant interferers. For instance, as shown in Fig. 1, the potential dominant interferers for users in sector 1 (shaded sector)/cell 4 , are the signal for users in sector $1 /$ cell 1 and sector $1 /$ cell $7^{1}$. Therefore, BS 4 sends the traffic information of users in sector $1 /$ cell 4 to BSs 1 and 7. Meanwhile, BS 4 receives the traffic information of users in sector $1 /$ cells 1 and 7 . This information includes the arrival time and service type of each packet waiting in the transmission queue. BSs 1, 4, and 7 use this information to avoid (or minimize) concurrent transmission of any users in these three sectors (sector $1 /$ cells 4,1 , and 7 ). We call these sectors that have potential dominant interferers "interference group". By eliminating the concurrent transmission within each interference group, the interference level can be drastically reduced. Obviously, there are other users that can cause interference to users in sector $1 /$ cell 4 including users in sector $1 /$ cells $2,3,5,6$, and 9 . However, their interference level is much less than that of sector $1 /$ cel1 1 and 7 because of the directional antennas used at both BSs and SSs.

Mathematically, the time slot allocation vector in sector $i /$ cell $j\left(S_{i j}\right)$ can be written as

$$
S_{i j}=\left[b_{i j}(0), b_{i j}(1), b_{i j}(2), \ldots, b_{i j}(8)\right],
$$

where $b_{i j}(k)$ is the $k^{\text {th }}$-slot indicator that takes the values 1 for a busy slot and 0 for an idle slot. For instance, the slot allocation vector in sector $1 /$ cell 4 is

$$
S_{14}=\left[b_{14}(0), b_{14}(1), b_{14}(2), \ldots, b_{14}(8)\right] \text {. }
$$

Since sector $1 /$ cells 1,4 , and 7 constitute an interference group, the algorithm ensures that the time slot allocation vectors $\left(S_{11}, S_{14}, S_{17}\right)$ are orthogonal.

The number of occupied slots in each sector and the decision of which time slots are allocated to which users in each sector depend on the employed intra-sector and intersector scheduling schemes. The intra-sector scheduling scheme schedules the packet transmission of all users inside a sector while the inter-sector scheme schedules the traffic transmission of different sectors within the

\footnotetext{
${ }^{1}$ Users in sector $1 /$ cell 7 cause this interference in the downlink because of the assumed wraparound grid.
} 
interference group as shown in Fig. 2. First Come First Serve (FCFS), Weighted Round-Robin (WRR), Weighted Fair Queuing (WFQ), Max-Min or any other schemes can be used at any of the two levels. For instance, with FCFS as the intra-sector and inter-sector scheduling scheme, the BS of each sector schedules its packet transmission based on their arrival time and the arrival time of packets in other sectors within the interference group. With WRR as the inter-sector scheduling scheme, each sector in the interference group is given portions of the time resources in a round-robin fashion with different weights to each class of service. The proposed technique is transparent in the sense that it can be integrated with any intra-sector and inter-sector scheduling schemes. Different scheduling schemes can be used at the two levels. For example, FCFS can be used as the intra-sector scheduling scheme while WRR is used at the inter-sector scheduling scheme.

It is apparent that the proposed algorithm reduces the number of available resources (time-slots) per sector, which can lead to a higher packet delay and a lower throughput. However, the throughput reduction is compensated by the enhancement of the signal quality so that the packet error rate is reduced and as a result the net throughput can be as high as the case without BS coordination or even higher. As for the packet delay increase, for non-real-time data services such as e-mail, ftp or http traffic, this increase in the packet delay can be tolerated as long as it is bounded and it leads to a better signal quality. For real-time services such as video and voice traffic, the condition of slotallocation vector orthogonality can cause high packet loss due to the increasing packet delay. In order to keep the packet loss at an acceptable value, each BS keeps track of the packet queuing delay. If it is found that the packet delay exceeds a certain threshold $\left(D_{t h}\right)$, a congestion flag is set and the algorithms relaxes the condition of slot allocation vector orthogonality within the intereference group of the congested users. Hence, the algorithm allows users in congestion to use all available time slots even if the potential dominant interferers are using the same slots. If the packet delay goes below $D_{t h}$, the algorithm returns to its original mode demanding the orthogonality of time slot allocation vectors of within each interference group.

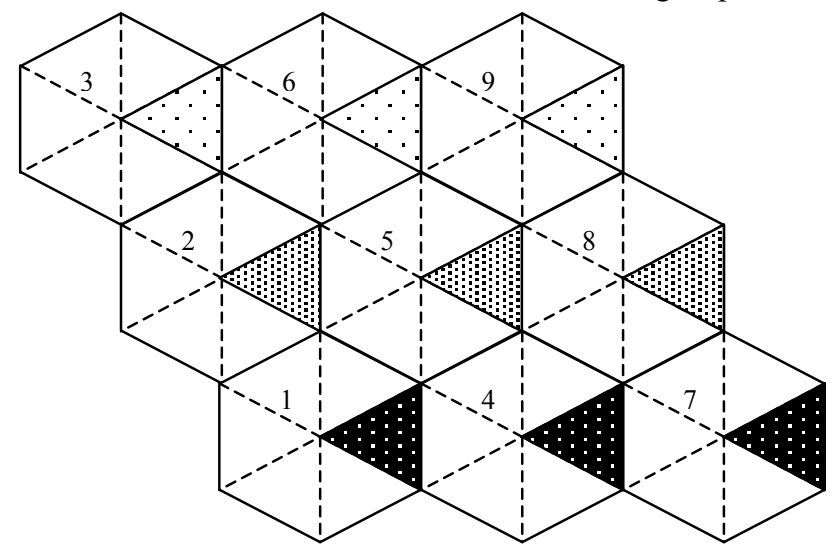

Fig. 1. Interference groups of sector 1.
Sector $1 /$ cell 1 transmission queue(s)

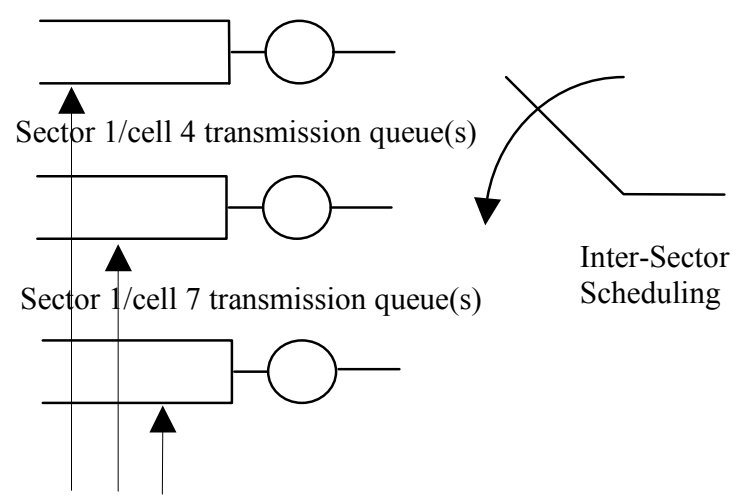

Intra-Sector

Scheduling

Fig. 2. Intra-sector and inter-sector scheduling.

\section{RESULTS}

The performance measures used to analyze the proposed algorithm are:

- Packet Error Rate (PER): A packet is considered in error if the SIR value is less than the targeted SIR level.

- $\quad$ Packet delay $(D)$ and packet delay jitter $\left(D_{j}\right)$ : 50 percentile is measured for both metrics.

- Packet loss $(P L)$ : A packet is considered as a lost packet if the packet delay $(D)$ exceeds the maximum delay of the real-time service $\left(D_{\max }\right)$.

- Throughput in packet per frame per sector: Total and net throughput values are measured. Erroneous transmitted packets are excluded in the net throughput calculation.

Computer simulation has been used for analyzing the system performance with BS coordination using the proposed algorithm and without BS coordination (intrasector scheduling only).

Figs. 3-5 depict the dependence of the performance metrics on the loading defined as the number of users per cell for non-real-time service (Internet traffic).

Fig. 3 shows that the proposed algorithm reduces the PER by almost one order of magnitude. For instance, at loading value of 150 user/cell, $P E R$ drops from $2.38 \times 10^{-2}$ without BS coordination to $2.2 \times 10^{-3}$ with the proposed algorithm. If the maximum tolerable $P E R$ is chosen to be equal to $2 \times 10^{-2}$, the loading value will be limited to $130 \mathrm{user} / \mathrm{cell}$ without BS coordination. With BS coordination using the proposed algorithm, PER does not exceed $1 \times 10^{-2}$ even with more than 400 user/cell.

Fig. 4 shows the net throughput for both cases. The total throughput of the proposed algorithm is slightly less than that of no BS Coordination. The total throughput is not plotted due to space limitation. Although the proposed algorithm slightly reduces the total throughput, the net throughput values of both cases are always very close since the gain from reducing $P E R$ due to the proposed algorithm 
compensates (and sometimes overweighs) the reduction in the total throughput.

The penalty of this performance enhancement is the increasing packet delay but only at high loading values as depicted in Fig. 5. At low to medium loading, the packet delay increase due to the proposed algorithm is insignificant. At high loading values (>330 user/cell), the packet delay of the proposed algorithm starts to increase exponentially. However, the packet delay is still in the acceptable range taking into account the delay tolerance of the non-real-time traffic. If the maximum packet delay is specified such that the 50-percentile should be less than $200 \mathrm{msec}$, the maximum number of users will be limited to 370 user/cell when the proposed algorithm is employed. This shows that the network capacity (or maximum loading) is delay-limited when the proposed algorithm is used while the network capacity is interference-limited if there is no BS coordination. The proposed algorithm slightly increases the packet delay jitter $\left(D_{j}\right)$. The 50percentile of the delay jitter is found to be constant and equal to $1.5 \mathrm{msec}$ with no $\mathrm{BS}$ coordination but it ranges from 1.5 to $4.4 \mathrm{msec}$ with the proposed algorithm.

Figs. 6-8 show the dependence of the performance metrics on the loading for real-time service (video traffic). The proposed algorithm is analyzed at the following four values for the delay threshold $\left(D_{t h}\right): 25,50,75$, and $100 \mathrm{msec}$.

As shown in Fig. 6, at low to medium loading values, PER is reduced by almost one order of magnitude; however at high loading values, PER is only reduced by almost $50 \%$. This is because at high loading values, the algorithm does not always keep the slot allocation vector orthogonality. It is evident that the value of $D_{t h}$ has no impact on PER values.

Fig. 7 shows that the proposed algorithm slightly increases the net throughput. As in the case of non-real-time traffic, the total throughput is slightly reduced due to the proposed algorithm. However, the net throughput is enhanced due to the significant reduction in PER.

From Fig. 8, it is apparent that the packet delay shows high dependence on the value of $D_{\max }$ particularly at high loading. For instance, at loading value of 30 user/cell, the 50 -percentile of packet delay $(D)$ is $28.2,53.1,78$, and $102.9 \mathrm{msec}$ for the four values of $D_{t h}$ mentioned above. It is evident that the proposed algorithm can limit the packet delay at (or slightly higher than) $D_{\max }$. All performance metrics, except the packet delay, does not show any dependence on $D_{t h}$. Therefore, it is better to choose a smaller value for $D_{t h}$ to have a smaller packet delay. As in the non-real-time traffic case, the proposed algorithm slightly increases the packet delay jitter $\left(D_{j}\right)$. The 50percentile of the delay jitter is found to be constant and equal to $1.2 \mathrm{msec}$ with no $\mathrm{BS}$ coordination but it ranges from 1.5 to $1.8 \mathrm{msec}$ with the proposed algorithm.

The packet loss $(P L)$ is found to be equal to zero for all cases even at high loading values.

\section{SUMMARY AND CONCLUSIONS}

A novel scheduling algorithm has been proposed for interference management in broadband wireless access networks. The proposed algorithm minimizes the concurrent transmission of potential dominant interferers using the orthogonality of the slot allocation vectors. The proposed algorithm can achieve lower PER and slightly better throughput at the expense of larger but acceptable packet delay. However, the increase in the packet delay can be tolerated in non-real-time services as long as it is bounded. With real-time services, the algorithm relaxes the condition of slot allocation vectors if the packet delay exceeds a certain threshold $\left(D_{t h}\right)$. The performance of various intra-sector and inter-sector scheduling schemes, with mixed traffic sources and with different classes of services are currently being investigated. Finding the optimum value for $D_{t h}$ that leads to the minimum packet delay with the lowest PER is a topic for future research.

\section{ACKNOWLEDGMENT}

This work is supported by the National Capital Institute of Telecommunication (NCIT), Ottawa, Ontario, Canada.

\section{REFERENCES}

[1] A. Solana and F. Palacio, "Scheduling and Quality of Service in W-CDMA," Proc. of ACT'99, pp.795-800, June 1999.

[2] K. Lee and M. El Zarki, "Scheduling Real-Time Traffic in IP-Based Cellular Networks," Proc. PIMRC'2000, pp. 12021206, Sept. 2000.

[3] M. Dillinger, J. Luo, and E. Schulz, "Radio Resource Scheduling Algorithms for Mixed VoIP and HTTP Traffic in HIPERLAN/2 System,” Proc. IST'2001, pp. 469-474.

[4] P. Viswanath, D. Tse, and R. Laroia, "Opportunistic Beamforming using Dumb Antennas," IEEE Trans. on Information Theory, vol. 48, No. 6, June 2002.

[5] X. Liu, E. Chong, and N. Shroff, "Transmission Scheduling for Efficient Wireless Utilization," Proc. IEEE Infocom '2001, pp. 776-786, April. 2001.

[6] M. Inoue, G. Wu, and Y. Hase, "Link-Adaptive Resource Scheduling for a Wireless Message Transport," Proc. IEEE Globecom'98, pp. 2223-2228, Nov. 1998.

[7] T. Fong, P. Henry, K. Leung, X. Qiu, N. Shankaranarayanan, "Radio Resource Allocation in Fixed Broadband Wireless networks, " IEEE Trans. on Communications, Vol. 46, No. 6, pp. 806-817, June 1998.

[8] K. Leung and A. Srivastava, "Dynamic Allocation of Downlink and Uplink Resource for Broadband Service in Fixed Wireless Networks," IEEE JSAC, Vol. 17, No. 5, pp. 990-1006, May 1999.

[9] L. Mailaender, H. Huang, and H. Viswanathan, "Simple Inter-cell Coordination Schemes for a High Speed CDMA Packet Downlink," Proc. IEEE VTC'00 Spring, Tokyo, Japan, May 2002.

[10] V. Erceg et al., "Channel models for fixed wireless applications," IEEE 802.16.3c-01/29r1, February 2001.

[11] C. Baugh and J. Huang, "Traffic model for 802.16 TG3 Simulations," IEEE 802.16.3c-01/53, March 2001.

[12] G. Cair, G. Taricco, and E. Biglieri, "Bit-interleaved coded modulation," IEEE Trans. on Info. Theory, vol. 44, no. 3, pp. 927-946, May 1998. 


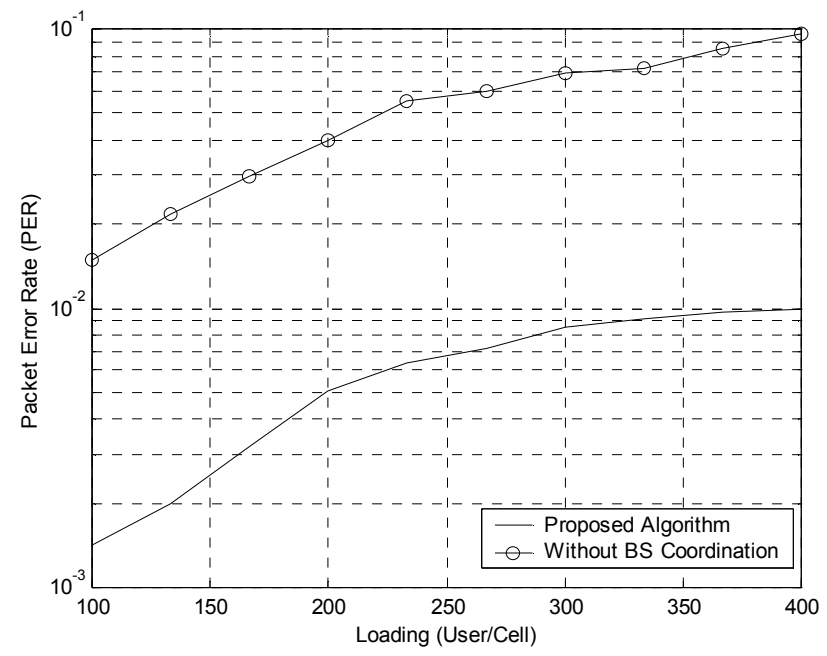

Fig. 3. Packet Error Rate of the Internet Traffic

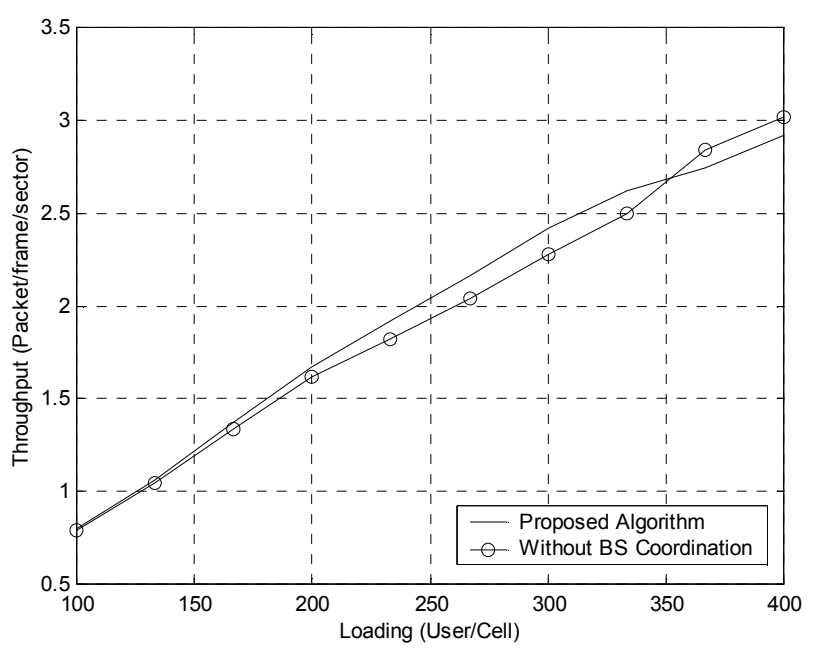

Fig. 4. Net Throughput of the Internet Traffic

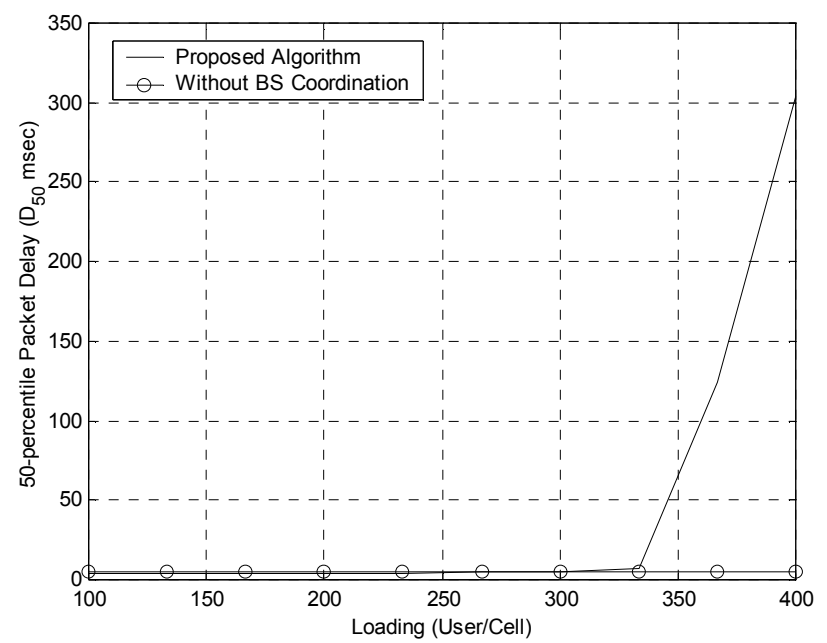

Fig. 5. 50-Percentile of Packet Delay of the Internet Traffic

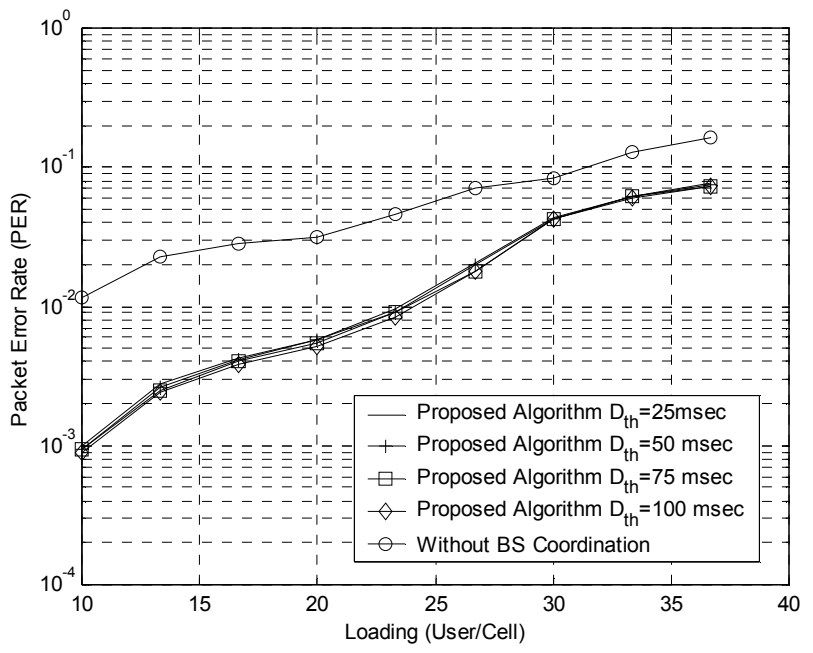

Fig. 6. Packet Error Rate of the Video Traffic

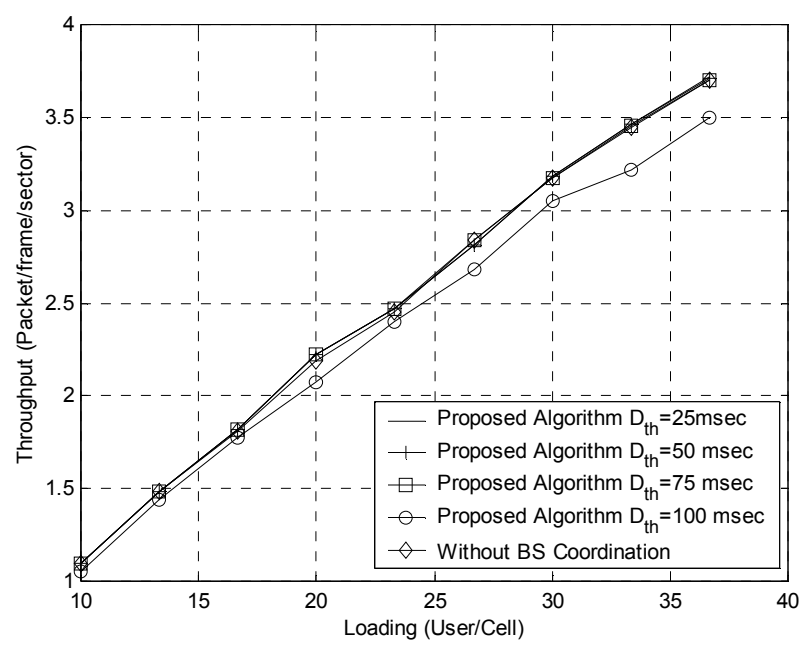

Fig. 7. Net Throughput of the Video Traffic

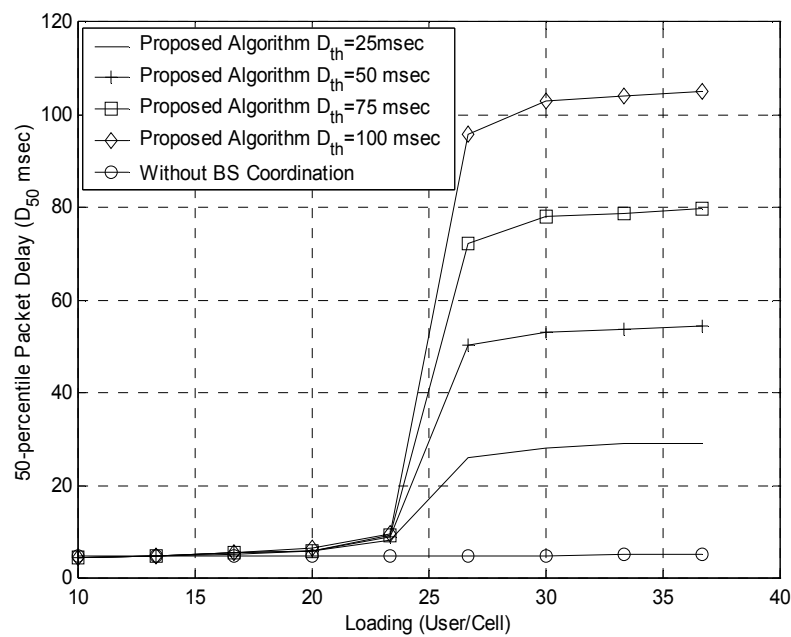

Fig. 8. 50-Percentile of Packet Delay of the Video Traffic 